\title{
Brachytherapy Seed Localization from Fluoroscopic Images Using a Statistical Classifier
}

\author{
Yi Su, Brian J. Davis, Michael G. Herman, Wayne N. LaJoie, and \\ Richard A. Robb \\ Mayo Clinic and Foundation, Rochester, MN 55905 USA
}

\begin{abstract}
A method to determine the number and location of seed images in clusters on a fluoroscopic image was developed based on a statistical analysis of cluster size in simulated fluoroscopic images. A modified 3-film technique was used to reconstruct the 3D positions of seeds from their image locations. Preliminary results suggest that this new method can identify all the seeds and localize them to an average precision of $0.55 \mathrm{~mm}$. This is an important step toward intraoperative radiation dose analysis.
\end{abstract}

\section{Introduction}

Permanent implantation of radioactive seeds is an effective and widely used treatment for prostate cancer. Further improvement of this procedure includes dose analysis in the operating room. One approach is to use intraoperative ultrasound-fluoroscopic image fusion in conjunction with the 3 -film seed reconstruction technique $[1,2]$. Here, we partition the seed localization process into three consecutive steps: 1) Segmentation of fluoroscopic images to get binary masks of seed images. 2) Determine the number and location of the seed images in each self-connected cluster. 3) Reconstruct the 3D seed positions using the seed image locations in different projections. The second step is an original contribution, whereas a modification of the well known 3-film technique is used for the third step.

\section{Method and Results}

We simulated X-ray projection images using computed tomography (CT) datasets from 10 prostate brachytherapy cases. The radiographic marker size of seven commonly used brachytherapy seeds was used to determine the size of the seed images on the projections. For each combination of seed location data and seed type, 181 projection images were generated with the projection angle ranging from -90 degrees to +90 degrees. Each projection image was segmented to generate a binary mask that contains all seed images, and then the pixel count for each self-connected cluster was obtained. Seed images that did not overlap with other seeds range from $56.9 \%$ to $92.9 \%$ in our simulation. The distribution of normalized pixel counts for clusters with 1 to 5 seeds from combination of seed location and seed type in one 
dataset was used to build our statistical classifier and tested on all combinations. For larger clusters the rounded number of the normalized pixel count was used as the seed number. The error rate of the classifier (incorrectly classified seed number divided by total seed number) was below 2 percent. The locations of the $n$ points that evenly divide the long axis of the minimum-enclosing rectangle of a cluster were then used as the locations of the centroids of the seed images, where $n$ is the number of seeds in the cluster. The reconstruction algorithm is based on the procedure reported by Narayanan et al. [1]. However, we modified the algorithm to handle cases where the number of seed images found in each projection is not the same. A cost function is used to optimize seed matching. The reconstruction result of one case with 154 implanted seeds and a marker size of $3.0 \mathrm{~mm} \times 0.5 \mathrm{~mm}$ is presented here. The average distance between the reconstructed seed location and true seed location was $0.55 \mathrm{~mm}$.

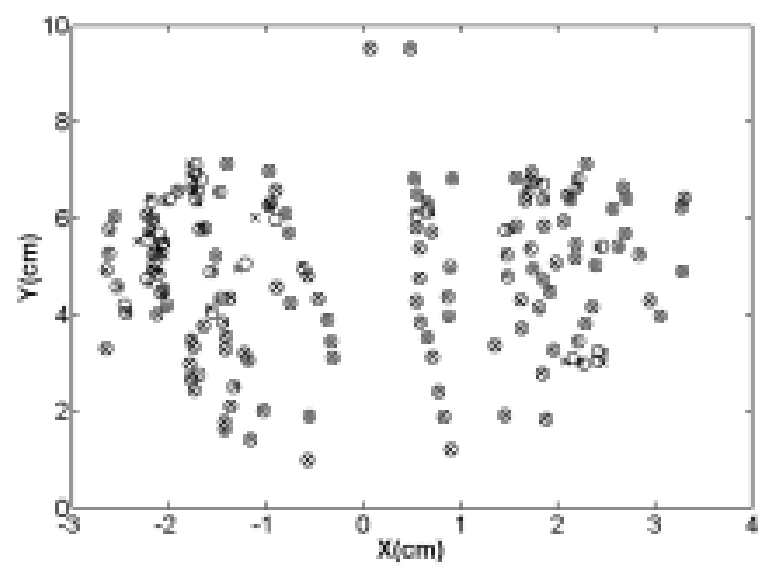

Fig. 1. Reconstructed seed position (o) compared to true seed position (x)

\section{Conclusion}

This study demonstrates that overlap of seed images on fluoroscopic projections is unavoidable in the current practice of permanent prostate brachytherapy. However, the cluster classification method as described here is highly effective in determining seed number and position in 3D. Future work will include phantom studies to further refine our algorithm. In addition, accurate ultrasound-fluoroscopic image registration methods are being developed to achieve intra-operative dose evaluation.

\section{References}

1. Narayanan S, Cho PS, Marks RJ II, Fast cross-projection algorithm for reconstruction of seeds in prostate brachytherapy. Med. Phys. Vol. 29(7), pp. 1572-1579, 2002

2. Gong L, et al. Ultrasonography and fluoroscopic fusion for prostate brachytherapy dosimetry. Int. J. Radiation Oncology Biol. Phys. Vol. 54 (5), pp.1322-1330, 2002 